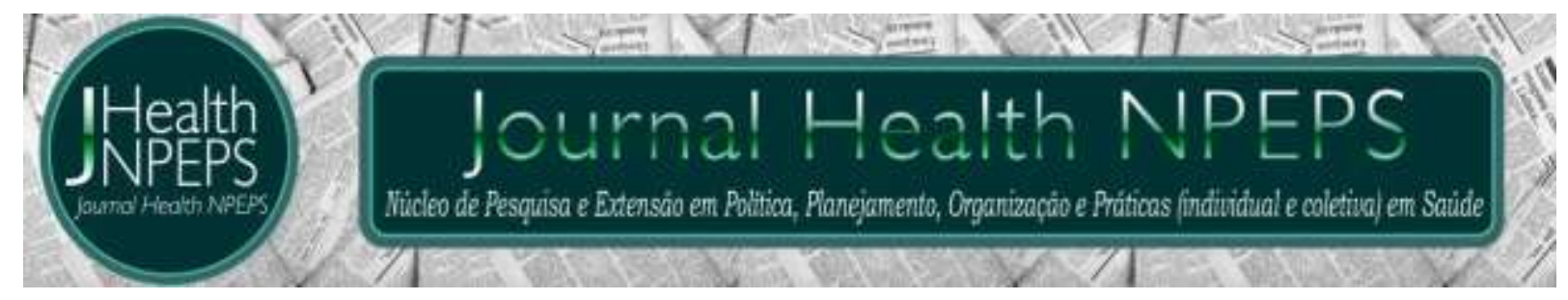

http://dx.doi.org/10.30681/2526101038233823

ARTIGO ORIGINAL

\title{
Características sociodemográficas e custo de hospitalizações por fratura de fêmur em idosos na Bahia
}

\section{Sociodemographic characteristics and cost of hospitalization for femur fracture in the elderly in Bahia}

\section{Características sociodemográficas y costo de hospitalización por fractura de fémur en ancianos em Bahía}

\section{Alana Oliveira Porto ${ }^{1}$, Carla Bianca de Matos Leal ${ }^{2}$, Marcela Andrade Rios ${ }^{3}$, Talitha Sonally Soares Fernandes ${ }^{4}$, Emanuella Soares Fraga Fernandes ${ }^{5}$, Ricardo Bruno Santos Ferreira ${ }^{6}$}

\section{RESUMO}

Objetivo: descrever os casos de internações por fratura de fêmur em idosos na Bahia entre os anos de 2012 e 2017, quanto às características sociodemográficas e o custo das hospitalizações. Método: trata-se de estudo ecológico e descritivo, realizado com dados de morbimortalidade hospitalar por fratura de fêmur em idosos na Bahia. Os dados foram coletados no sistema de informação hospitalar, disponibilizados pelo departamento de informática do Sistema Único de Saúde.

\footnotetext{
${ }^{1}$ Enfermeira. Especialista. Residente em Saúde da Família pela FESF/Fiocruz. Universidade Estadual da Bahia (UNEB). Salvador, Bahia, Brasil. E-mail: alana.udi20@hotmail.com ORCID ID: https://orcid.org/0000-0001-8922864X Autor principal - Endereço para correspondência: Rua José Valter Reis, 178, DCR. Urandi-BA.

${ }^{2}$ Enfermeira. Especialista. Docente no curso técnico em enfermagem no Centro de Estudo Técnico Criando Talentos - CETCT. Universidade do Estado da Bahia (UNEB) - Campus XII. Guanambi, Bahia, Brasil. E-mail: carlabiancagbi@hotmail.com ORCID ID: https://orcid.org/0000-0002-5090-4863

${ }^{3}$ Enfermeira. Doutoranda em Enfermagem e Saúde pelo PPGES/UESB. Docente do curso de Bacharelado em Enfermagem da Universidade do Estado da Bahia (UNEB). Universidade do Estado da Bahia - Campus XII. Guanambi, Bahia, Brasil. E-mail: marcelariosenf@gmail.com ORCID ID: https://orcid.org/0000-0001-7180-2008 ${ }^{4}$ Enfermeira. Mestranda em Enfermagem pela Universidade Estadual de Feira de Santana (UEFS). Enfermeira do Hospital Regional de Guanambi. Universidade do Estado da Bahia (UNEB) - Campus XII. Guanambi, Bahia, Brasil. E-mail: talithasonally@gmail.com ORCID ID: https://orcid.org/0000-0001-5868-8651

${ }^{5}$ Enfermeira. Mestre em Saúde Coletiva pela Universidade Estadual de Feira de Santana (UEFS). Professora auxiliar do curso de enfermagem da Universidade do Estado da Bahia (UNEB) - Campus XII. Guanambi, Bahia, Brasil. E-mail: manusff@yahoo.com.br ORCID ID: https://orcid.org/0000-0003-0614-4817

${ }^{6}$ Enfermeiro. Mestre em Enfermagem pela Universidade Estadual de Feira Santana (UEFS). Docente substituto na Universidade do Estado da Bahia (UNEB) - Campus XII. Guanambi, Bahia, Brasil. E-mail: ricardobrunoenf@gmail.com ORCID ID: https://orcid.org/0000-0003-0614-4817
}

Este artigo está licenciado sob forma de uma licença Creative Commons Atribuição 4.0 Internacional, que permite uso irrestrito, distribuição e reprodução em qualquer meio, desde que a publicação original seja corretamente citada. 
Realizou-se cálculos de frequência relativa e coeficiente de letalidade. Resultados: houve 11.941 internações por fratura de fêmur em idosos nesse período. Verificaram-se, em sua maior parte, hospitalizações e óbitos prevalentes em mulheres, maiores de 80 anos e de cor ignorada, com taxa de letalidade de $4,4 \%$. Conclusão: sugere-se a partir da ascensão do índice de hospitalizações por fratura de fêmur na Bahia, a necessidade de reforçar políticas públicas voltadas para o idoso, a fim de garantir maior acesso à saúde, melhor qualidade de vida e menor prejuízo social.

Descritores: Epidemiologia; Saúde do Idoso; Hospitalização; Qualidade de vida; Saúde Pública.

\section{ABSTRACT}

Objective: to describe the cases of hospitalization for femur fracture in the elderly in Bahia between 2012 and 2017, regarding sociodemographic characteristics and the cost of hospitalizations. Method: this is an ecological and descriptive study, performed with data on hospital morbidity and mortality due to femur fracture in the elderly in Bahia. Data were collected through the Hospital Information System, made available by the Information System Department of the Unified Health System. Relative frequency and lethality coefficient calculations were performed. Results: there were 11,941 hospitalizations for femur fracture in the elderly, corresponding to $0.31 \%$ of general hospitalizations during this period, excluding deliveries. Most of the hospitalizations and deaths observed were prevalent among women, 80 years and over and of unknown skin color, with a lethality rate of $4.4 \%$. Conclusion: based on the rise in the hospitalization rate for femur fracture in Bahia, the need to reinforce public policies aimed at the elderly in order to ensure greater access to health, better quality of life and lower social impairment is suggested.

Descriptors: Epidemiology; Health of the Elderly, Hospitalization; Quality of Life; Public Health.

\section{RESUMEN}

Objetivo: describir los casos de hospitalización por fractura de fémur en ancianos en Bahía entre 2012 y 2017, en relación con las características sociodemográficas y el costo de las hospitalizaciones. Método: este es un estudio ecológico y descriptivo, realizado con datos sobre morbilidad y mortalidad hospitalaria por fractura de fémur en ancianos en Bahía. Los datos se recopilaron a través del Sistema de información del hospital, puesto a disposición por el Departamento del sistema de información del Sistema de salud unificado. Se realizaron cálculos de frecuencia relativa y coeficiente de letalidad. Resultados: hubo 11.941 hospitalizaciones por fractura de fémur en ancianos, lo que corresponde al 0,31\% de las hospitalizaciones generales durante este período, excluyendo los partos. Se observaron la mayoría de las hospitalizaciones y muertes prevalentes entre mujeres mayores de 80 años y de color desconocido, con una tasa de letalidad del 4,4\%. Conclusión: desde el aumento de la tasa de hospitalización por fractura de fémur en Bahía, se sugiere la necesidad de reforzar las políticas públicas dirigidas a los ancianos para garantizar un mayor acceso a la salud, una mejor calidad de vida y un menor deterioro social.

Descriptores: Epidemiiología; Salud del Ancianos; Hospitalización; Calidad de Vida; Salud Pública. 


\section{INTRODUÇÃO}

O avanço da medicina tem
possibilitado o aumento da
expectativa de vida a partir da
melhoria na qualidade de vida da
população, no entanto, atrelado à
longevidade, $\quad$ verifica-se
acometimento de condições clínicas
inerentes à faixa etária idosa ${ }^{1,2}$.

Ao considerar 0 termo morbidade como a soma de agravos de uma dada população, usa-la para estimar quantitativamente doenças em uma população permite inferir sobre as condições de saúde da mesma ${ }^{3}$ Observou-se nesta perspectiva, que no Brasil, as cinco principais morbidades prevalentes em internações de idosos dentre os 21 capítulos da classificação internacional de doenças são doenças do aparelho respiratório, neoplasias, doenças do aparelho digestório e causas externas, consecutivamente ${ }^{4}$.

No que tange as causas externas, as quedas apresentam-se como um evento frequente, recorrente e multifatorial na referida faixa etária e que traz consequências negativas ao idoso, como a ocorrência de fraturas, sendo a fratura de fêmur mais prevalente ${ }^{4,5}$.

A fratura de fêmur é considerada um grave problema de saúde, principalmente em idosos. Visto que esse grupo, em sua maioria, possui alguma debilidade, logo, uma fratura dessa magnitude potencializa suas limitações, dependências, perda de autonomia, diminui as chances de um bom prognóstico, em virtude de procedimentos cirúrgicos e recorrentes hospitalizações, além de onerar os cofres públicos $1,2,6,7$.

Além dos prejuízos ao idoso, a fratura de fêmur pode ser muito dispendiosa para a família, tendo em vista que comumente esta assume o papel de cuidadora e na maioria das vezes não recebe remuneração ou treinamento profissional, o que pode influenciar na reabilitação do idoso ${ }^{8}$.

No país, apenas no ano de 2017 foram registradas 54.986 internações por fratura de fêmur em idosos, com valores crescentes em relação aos anos anteriores, o que chama atenção para a necessidade de medidas preventivas de trauma em idosos ${ }^{4}$.

É válido salientar que as pesquisas nacionais não apresentam 
valores reais de incidência de fratura de fêmur no Brasil, tendo em vista a possibilidade de subnotificação, seja por deficiência na alimentação do sistema ou pelos casos de fratura não atendidos, o que remete a valores possivelmente maiores ${ }^{9}$. No panorama de uma hospitalização a idade avançada, incapacidades física e cognitiva pré-existentes, polifarmácia, comorbidades e histórico de quedas são considerados fatores que contribuem para a perda funcional de maioria dos idosos ${ }^{10}$, o que atualmente se denomina Incapacidade Associada

à Hospitalização (IAH), e por sua vez, além de interferir na independência e qualidade de vida do idoso, ainda é preditor para maiores gastos e óbito ${ }^{11}$.

Ao considerar a crescente incidência de fratura de fêmur em idosos, surgiu a seguinte questão norteadora: Qual o perfil de internação e o impacto gerado pelas fraturas de fêmur em idosos, na Bahia? Diante o exposto, este estudo objetiva descrever os casos de internações por fratura de fêmur em idosos na Bahia entre os anos de 2012 e 2017, quanto às características sociodemográficas e o custo das hospitalizações.

\section{MÉTODO}

Trata-se de um estudo descritivo, do tipo ecológico, realizado com base em dados de morbimortalidade hospitalar por fratura de fêmur em idosos na Bahia, entre os anos de 2012 a 2017. Os dados foram obtidos no segundo semestre de 2018 por meio do Sistema de Informação Hospitalar (SIH), disponibilizados eletronicamente e consolidados no Departamento de Informática do Sistema Único de Saúde (DATASUS).

Foram excluídos os casos notificados ao $\mathrm{SIH}$ e registrados como causa da internação segundo a Classificação Internacional de Doenças, em sua $10^{a}$ revisão (CID-10), compreendendo apenas fraturas de fêmur (código S72), dentre as demais fraturas.

Foram estudadas as seguintes variáveis: sexo, cor, faixa etária, considerando acima de 60 anos de idade, tipos de fratura, média de permanência, valor médio e total de hospitalização, óbitos por internação e macrorregiões de saúde. Tabularamse os dados com auxílio do Microsoft Office Excel onde se realizaram 
cálculos das frequências absolutas e relativas.

0 coeficiente de letalidade foi calculado por meio da divisão do número de óbitos hospitalares por fratura de fêmur em idosos na Bahia pelo total de casos hospitalizados por tal condição clínica no período investigado e multiplicado por 100. Por se tratar de um estudo com dados secundários e de domínio público, a apreciação do Comitê de Ética em Pesquisa é dispensável.

\section{RESULTADOS}

Foram registrados no SIH/SUS 11.941 casos de internação por fratura de fêmur, correspondendo a $0,31 \%$ dos casos de internação geral nesse período, excluindo-se internações por partos. Verificou-se aumento progressivo destas internações, sobretudo no sexo feminino (Figura 1).

Figura 1 - Evolução de internações por fratura de fêmur em idosos por sexo na Bahia nos anos de 2012 a 2017.

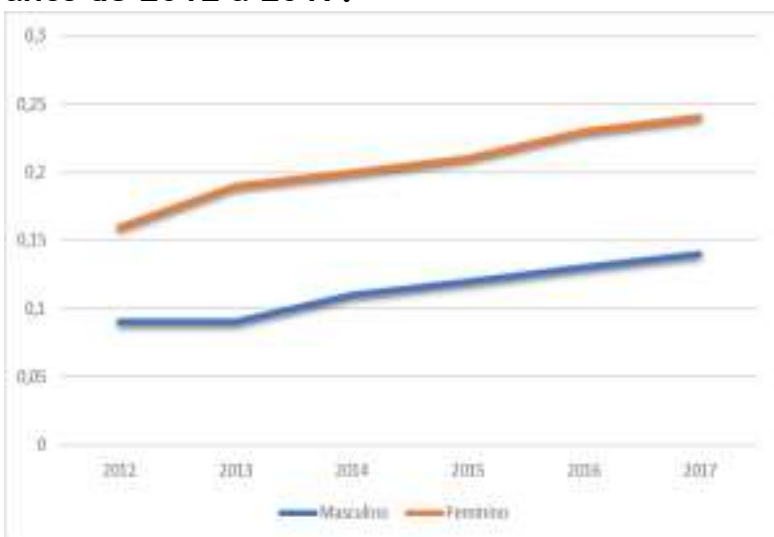

Fonte: SIH/DATASUS, 2019.
Fratura de fêmur ocupa a segunda posição entre as causas de internação por fraturas em idosos, representando $40,6 \%$ dos casos, sendo a primeira posição ocupada por fratura de outros ossos dos membros $42,3 \% \quad(n=12.426)$. Outros tipos de fratura como a de pescoço, tórax ou pelve e fratura de osso de pelve apresentaram percentual inferior a $2,7 \%(n=794)$.

Quanto à cor, não foi possível traçar o perfil para esta variável, tendo em vista a subnotificação desta informação. Quanto à faixa etária, é consideravelmente maior a prevalência de fraturas em idosos com idade mais avançada (Tabela 1).

Tabela 1 - Perfil sociodemográfico de internação e óbitos em idosos por fratura de fêmur na Bahia entre os anos 2012 e 2017.

\begin{tabular}{lcccccc}
\hline \multicolumn{1}{c}{ Perfil } & \multicolumn{2}{c}{$\begin{array}{l}\text { Intern. s/ } \\
\text { óbito }\end{array}$} & \multicolumn{2}{c}{$\begin{array}{c}\text { Intern. } \\
\text { c/ óbito }\end{array}$} & \multicolumn{2}{c}{ Total } \\
\hline Sexo & $\mathbf{n}$ & $\%$ & $\mathbf{n}$ & $\%$ & $\mathbf{n}$ & $\%$ \\
Feminino & 73 & 64,4 & 33 & 64,6 & 7686 & 64,4 \\
& 49 & & 7 & & & \\
Masculino & 40 & 35,6 & 18 & 35,4 & 4255 & 35,6 \\
& 70 & & 5 & & & \\
Cor & 57 & 5,0 & 29 & 5,5 & 606 & 5,1 \\
Branca & 7 & & & & & \\
& 15 & 1,4 & 8 & 1,5 & 164 & 1,4 \\
Preta & 6 & & & & & \\
& 32 & 28,3 & 12 & 23,5 & 3353 & 28,0 \\
Parda & 30 & & 3 & & & \\
Amarela & 12 & 1,1 & 4 & 0,7 & 129 & 1,1 \\
Indígena & 5 & & & & & \\
& 1 & 0,0 & - & 0,2 & 1 & 0,0 \\
Sem & 73 & 64,2 & 35 & 68,6 & 7688 & 64,4 \\
Informação & 30 & & 8 & & & \\
\hline
\end{tabular}




\begin{tabular}{lllcccc}
\hline Faixa etária & & & & & & \\
$60-69$ anos & 25 & 22,2 & 60 & 11,5 & 2601 & 21,8 \\
& 41 & & & & & \\
$70-79$ anos & 35 & 31,1 & 10 & 20,7 & 3655 & 30,6 \\
& 47 & & 8 & & & \\
80 e mais & 53 & 46,7 & 35 & 67,8 & 5685 & 47,6 \\
& 31 & & 4 & & & \\
\hline
\end{tabular}

Fonte: SIH/DATASUS, 2019.

Foram estudados também os óbitos a partir das hospitalizações da referida condição clínica e, considerando-se que ocorreram 522 mortes, pode-se verificar que a taxa de letalidade de fratura de fêmur em idosos foi de 4,4\%. As características sociodemográficas revelaram que 64,6\% ( $n=337)$ eram mulheres, assim como a maior frequência de óbitos, a letalidade $(2,8 \%)$ também se apresentou superior em detrimento ao masculino $(1,5 \%)$.

Em relação à cor para os casos de óbitos, 68,6\% (n=358) não apresentaram informações, seguido da prevalência da cor parda 23,5\% $(n=123)$. Quanto à faixa etária, 67,8\% $(n=354)$ dos óbitos ocorreram entre 80 anos e mais, seguido por 70-79 referente a 20,7\% (n=108) e 60 a 69 anos, que corresponde a $11,5 \%(n=60)$.

Ao analisar a letalidade desses casos, observou aumento com 0 avançar da idade, apresentando 2,3\% para aqueles com idade entre 60-69 anos; $2,9 \%$ entre $70-79$ anos e 6,2\% para idosos acima de 80 anos.
Constatou-se que a média de dias de permanência desses idosos foi aproximadamente 9,1 dias, sendo que o sexo feminino apresentou 9,3 dias, enquanto que as internações do sexo masculino duraram em torno de 8,9 dias. Quanto ao valor médio de internação, verificou-se em 2017 o valor mais alto, $\mathrm{R} \$ 2453,62$; já em 2012, este foi o menor de todos com $\mathrm{R} \$ 1987,94$ por internação (Tabela 2).

Tabela 2 - Valor médio de internação hospitalar por fratura de fêmur em idosos na Bahia entre os anos 2012 e 2017.

\begin{tabular}{cccc}
\hline Ano & Atendimentos & $\begin{array}{c}\text { Valor } \\
\text { médio } \\
\text { R\$ }\end{array}$ & $\begin{array}{c}\text { Valor } \\
\text { total R\$ }\end{array}$ \\
\hline 2012 & 1.697 & $1.987,94$ & 3.373 .533 \\
2013 & 1.810 & $2.172,20$ & 3.931 .680 \\
2014 & 2.047 & $2.184,00$ & 4.470 .655 \\
2015 & 2.086 & $2.176,27$ & 4.539 .689 \\
2016 & 2.218 & $2.290,80$ & 5.080 .997 \\
2017 & 2.083 & $2.453,62$ & 5.110 .897 \\
TOTAL & 11.941 & $2.210,80$ & 26.507 .451 \\
\hline Fonte: SIH/DATASUS, 2019.
\end{tabular}

Em relação às internações hospitalares por macrorregião, a região Leste destaca-se em relação às demais, pois apresentou os maiores números de internações em todos os anos 38,2\% (n=4566). A macrorregião Centro Norte retratou os menores índices com 3,7\% $(n=344)$ internações.

\section{DISCUSSÃO}

Com envelhecimento humano e maior expectativa de vida, 
as causas externas não intencionais tornaram-se mais frequentes e têm assumido papel importante dentre os problemas de saúde pública no Brasil $^{12}$. Nesta perspectiva, destaca-se a fratura de fêmur, lesão comumente relacionada à queda da própria altura, que se associa neste estudo ao elevado número de internações da população idosa, com crescimento significativo ao decorrer dos anos estudados, além do mais apresenta o potencial de gerar impactos negativos ao idoso em todas as suas nuances: físico, psicológico e social ${ }^{1,7}$.

Em Florianópolis, foi realizado um estudo que revelou as quedas no domicílio seguido de fratura de membros como principal motivo para entrada no atendimento de emergência por causas externas em idosos $^{12}$.

A ocorrência de quedas acima dos 60 anos de idade é considerada efeito do processo de senescência para alguns estudiosos que citam a instabilidade postural, acuidades visual e auditiva prejudicadas, distúrbio de equilíbrio, marcha e coordenação motora como principais causas. No entanto, tem sido alta a ocorrência de quedas por fatores externos como o uso de medicamentos psicotrópicos, ambiente sem acessibilidade e com obstáculos em locais de circulação ${ }^{7,13}$.

Independente da causa, ainda na perspectiva de quedas, este episódio não pode ser considerado um evento normal do envelhecimento ${ }^{3}$. Sabe-se que sua ocorrência produz perda de autonomia, dependência, danos financeiros, sociais, emocionais e mentais, bem como aumento da morbidade e mortalidade, logo, são imprescindíveis medidas preventivas que envolvam os profissionais de saúde em todos os níveis de assistência, garantindo atendimento multiprofissional, integral e longitudinal ao idoso ${ }^{13}$.

Algumas medidas de custobenefício favoráveis são fortemente propagadas para a redução de quedas em idosos, dentre elas destacam-se o incentivo à redução de uso de medicamentos psicotrópicos, tratamento adequado para doenças sistêmicas e cognitivas, prática regular de exercícios físicos objetivando o aumento de força muscular e melhora na postura e equilíbrio e por fim, a acessibilidade no ambiente domiciliar que diz respeito à eliminação de objetos/tapetes espalhados na 
superfície, instalação de corrimão em rampas e escadas e uso de pisos antiderrapantes ${ }^{14}$.

A elevada incidência de fraturas de fêmur deve-se também a osteoporose, uma doença silenciosa, altamente prevalente em idosos e caracterizada pela deficiência no metabolismo do osso, que por sua vez vulnerabiliza a microarquitetura deste, propiciando maior risco a fraturas $6,15,16$. Diante do risco, é válido o desenvolvimento de medidas profiláticas, alimentação rica em frutas e verduras, exercícios físicos, ingesta de suplementos de cálcio e vitamina $D$ e uso de bifosfonatos conforme prescrição profissional ${ }^{15-17}$.

Em relação ao tipo de fratura, corroborando com este estudo, pesquisa também apontou os membros como locais mais atingidos, e as quedas como a causa predominante para fraturas ${ }^{12}$.

Em se tratando do sexo, foi verificado maior prevalência de fratura em mulheres, o que é verificado em outros estudos 1,6,7,9,12. Essa variável pode ser justificada pelas atividades domésticas exercidas pelas mulheres mesmo na terceira idade, maior aderência a atividades de lazer, além de fatores fisiológicos como perda de massa e força muscular após menopausa ${ }^{6,7,13}$. Além disso, pesquisadores verificaram que as fraturas têm ocorrido com maior frequência durante o dia, sendo esta variável compatível com a maior incidência de fraturas em mulheres, diante das atividades domésticas realizadas ${ }^{7,12}$.

Quanto à cor, não foi possível traçar o perfil devido ausência de registro desta informação, diante disso, observa-se a deficiência dos profissionais responsáveis pela alimentação do sistema de informação do SUS, ao considerarem esta variável irrelevante. Ainda em relação à cor, considerando os dados informados, os pardos encontram-se em primeira colocação para fratura de fêmur em idosos. Possivelmente, justifica-se esse dado pelo processo de miscigenação pelo qual passou o Brasil ao longo de sua história ${ }^{18}$. Ademais a região Nordeste, em especial na cidade de Salvador, capital da Bahia, concentra-se uma parcela importante da população negra e parda deste país ${ }^{7}, 18$.

No que se refere à idade, verificou-se neste estudo maior prevalência de fratura de fêmur em idosos em maiores de 80 anos, 
corroborada por estudos sobre hemiartroplastia do quadril pós fratura de fêmur em idosos, em que a média de idade foi de 83 anos ${ }^{1,19}$.

Embora haja estudos que apresentem predominância em faixas etárias inferiores (igual ou maior que 70 anos), há relatos de que maior risco de fratura decorrente ao avanço da idade, justificado pelo fato de que na população senil o quadril absorve maior força do impacto e sendo esta absorção crescente e linear à fragilidade óssea presente no processo de envelhecimento, qualquer trauma de baixa energia pode provocar uma lesão ${ }^{1,6,7}$. Ademais, diante das iniciativas visando a envelhecimento saudável, é possível observar idosos em boa forma e contribuindo ativamente na comunidade, o que aumenta sua exposição ao risco ${ }^{12}$.

A terapêutica escolhida para tratar um paciente com fratura de fêmur baseia-se primeiramente na análise da condição clínica que diz respeito aos fatores que possam interferir na recuperação do mesmo, como doenças preexistentes, estado mental, idade e nível de atividade; e em seguida, é avaliado se a uma fratura é estável ou instável, logo, pacientes críticos como idosos, com fratura instável são submetidos a procedimentos cirúrgicos ${ }^{1,6}$.

A qualidade de vida do idoso é um fator importante para se avaliar após o tratamento cirúrgico de uma fratura associada à alta taxa de mortalidade ${ }^{6}$. Realizou-se em Manaus um estudo cujo objetivo era avaliar a qualidade de vida de idosos submetidos à cirurgia no qual se revelou que após 3 meses da realização do procedimento, a capacidade funcional é significantemente menor quando comparada ao período anterior, além de haver redução do aspecto físico e emocionais, bem como haver presença de dor ${ }^{1}$.

Ao analisar os óbitos, foi revelado neste estudo importante letalidade para idosos com fratura de fêmur, sobretudo em mulheres e na faixa etária de 80 anos acima, sendo estas as mesmas características sociodemográficas apresentadas nas hospitalizações. 0 resultado apresentado condiz com a literatura em que as mulheres são mais propensas ao desfecho de morte após a ocorrência de uma fratura de fêmur, sendo este evento mais frequente conforme o avanço da idade, sobretudo quando o paciente possui 
distúrbios

cardiopulmonares, metabólicos, renais ou acidente vascular cerebral 6,9 .

Houve divergência entre 0 tempo de hospitalização deste estudo e outra publicação em que a média de permanência verificada na capital da Bahia teve como média 14 dias $^{6}$. Já no âmbito nacional, a média de permanência foi de apenas sete dias sendo este curto período de internação associado à deficiência na continuidade do cuidado, haja vista que fratura de ossos longos em idosos necessitam além do procedimento cirúrgico, tratamento voltado para o fator que desencadeou a lesão, prevenindo novos eventos ${ }^{9}$.

Quanto aos custos econômicos, de 2012 a 2017 houve aumento médio de $\mathrm{R} \$$ 465,68 por internação na Bahia, logo, verifica-se o crescente desfalque no orçamento público voltado para as internações por fratura de fêmur, sobretudo em idosos devido à alta incidência.

São poucos estudos que trazem reflexão de gastos por Estado, diante disso, ao considerar as hospitalizações no âmbito nacional, estudo revelou gastos de $\mathrm{R} \$$ 43.564.378,10, apenas no ano de 2008, representando cerca de $2 \%$ do valor total de internações em idosos 9 . Em 2017, nove anos após, o gasto foi de $\mathrm{R} \$ 138.580 .543,81$, o que representa $2,7 \%$ do gasto por hospitalizações gerais nessa população e cerca de três vezes a mais o que o valor apresentado em $2008^{4}$.

Os valores supracitados refletem bem o impacto financeiro gerado a partir da referida condição. Ademais, vale ressaltar os prejuízos na organização dos familiares durante o processo de reabilitação de seus entes, seja com medicamentos, cuidadores e até mesmo perda da renda ao se dedicar exclusivamente aos cuidados ${ }^{8}$.

No que diz respeito às macrorregiões da Bahia, não observou na literatura justificativa referente à maior incidência de hospitalizações na região Leste. Considerando o crescente número de internações por fratura de fêmur em idosos, presente em todos os anos investigados, sugerese a realização de estudos que busquem elucidar as causas que levaram a tal resultado, possibilitando assim, a implantação de programas preventivos de quedas e fraturas nesta população.

$$
\text { Entre as limitações }
$$

encontradas na elaboração deste 
estudo, cita-se a subnotificação de informações no DATASUS, no entanto, as informações ali disponíveis permitem identificar os agravos mais prevalentes na população senil e a partir de então, investigar os fatores associados a estes agravos e traçar medidas preventivas e de proteção à saúde do idoso, bem como redução do impacto econômico.

\section{CONCLUSÃO}

Este estudo possibilitou verificar crescente índice de morbimortalidade hospitalar por fratura de fêmur em idosos na Bahia, sendo estes eventos mais frequentes em mulheres, maiores de 80 anos, com aproximadamente nove dias de internação e custo médio de R\$ 2210,80 nos anos estudados.

No Brasil existem projetos voltados para prevenção de fratura em idosos, no entanto, ao considerar o elevado número de lesões ocorridas, as consequências econômicas, sociais e psicológicas advindas dessa condição e principalmente os desfechos de morte, torna-se necessário reforçar e ampliar as políticas públicas direcionadas para o público idoso, a fim de obter declínio nos índices de fraturas, melhor qualidade de vida e acesso aos serviços de saúde e, por conseguinte, redução de gastos gerados.

Nessa perspectiva, é válido ressaltar a importância dos profissionais de saúde na avaliação das restrições do idoso, uso de medicamentos, condições de moradia, enfim, verificar todos os fatores de risco para queda ou aquisição de fraturas a fim de traçar planos de prevenção para este agravo.

\section{REFERÊNCIAS}

1. Abreu EL, Oliveira MHA. Avaliacão da qualidade de vida dos pacientes submetidos à hemiartroplastia do quadril. Rev Bras Ortop. 2015; 50(5): 530-536.

2. Guimarães FAM, Belangero WD. Fatores de risco associados ao evento da fratura transtrocanteriana do fêmur. Rev Bras Med. 2014; 71(10).

3. Girondi JBR, Nothaft SCS, Santos SMA, Oliveira F, Sebold LF, Kempfer SS. Estudo do perfil de morbimortalidade entre idosos. Rev Enferm UFSM. 2013; 3(2):197-204.

4. DATASUS. Ministério da Saúde. Informações de Saúde, 
Epidemiológicas e Morbidade. 2018.

Disponível

em:

http:// datasus.saude.gov.br.

Acesso em: 31 Mai 2019.

5. Cruz DT, Cruz FM, Chaoubah A, Leite ICG. Fatores associados a quedas recorrentes em uma coorte de idosos. Cad Saúde Colet. 2017; 25(4): 475-482.

6. Conceição AM, Filho FCG, Dias JP. Internações por fraturas de fêmur em Salvador, Bahia. Rev Baiana Saúde Pública. 2016; 40(2): 298314.

7. Santana DF, Reis HFC, Ezequiel DJS, Ferraz DD. Perfil funcional, sociodemográfico e epidemiológico de idosos hospitalizados por fratura proximal de fêmur. Rev Kairós. 2015; 18(1): 217-234.

8. Rocha SA, Avila MAG, Bocchi SCM. Influência do cuidador informal na reabilitação do idoso em pósoperatório de fratura de fêmur proximal. Rev Gaúcha Enferm. 2016; 37(1): e51069.

9. Bortolon PC, Andrade CLT, Andrade CAF. O perfil das internações do SUS para fratura osteoporótica de fêmur em idosos no Brasil: uma descrição do triênio 2006-2008. Cad Saúde Pública. 2011; 27(4):733-742.
10. Bouxsein ML, Ealstell R, Lui LY, Wu LA, de Papp AE, Grauer A, et al. Change in Bone Density and Reduction in Fracture Risk: A Meta-Regression of Published Trials. J Bone Miner Res. 2019 Apr; 34 (4): 632-642.

11. Carvalho TC, Valle AP, Jacinto AF, Mayoral VFS, Villas-Boas PJF. Impact of hospitalization on the functional capacity of the elderly: A cohort study. Rev bras geriatr gerontol. 2018; 21(2): 134-142.

12. Felipe F, Mesadri PS, Teixeira S, Santos J, Pelegrini A. Atendimentos de emergência por causas externas não intencionais envolvendo idosos de Florianópolis, SC: VIVA 2006-2007. Estud interdiscipl envelhec. 2016; 21(1):235-248.

13. Caberlon IC, Bós AJG. Diferenças sazonais de quedas e fraturas em idosos gaúchos. Ciênc Saúde Coletiva. 2015; 20(12):3743-3752.

14. Soares DS, Mello LM, Silva AS, Nunes AA. Análise dos fatores associados a quedas com fratura de fêmur em idosos: um estudo caso-controle. Rev Bras Geriatr Gerontol, 2015; 18(2):239-248.

15. Nobre AC, Vieira VMSF, Nobre AC, Pamplona YAP. Caracterização do 
uso do alendronato para osteoporose na Atenção Primária à Saúde. Rev Bras Med Fam Comunidade. 2016; 11(38): 1-9.

16. Gosling T, Krettek C. Femurschaftfraktur.

Unfallchirurg. 2019; 122:59.

17. Silva PZ, Schneider RH. O papel da vitamina $D$ na força muscular em idosos. Acta Fisiatr. 2016; 23(2):96-101.

18. Instituto Brasileiro de Geografia e Estatística - IBGE [Internet]. Distribuição Espacial da População Segundo Cor ou Raça. 2010.
Disponível em: https://www.ibge.gov.br/geocien cias-novoportal/cartas-emapas/sociedade-eeconomia/15963-distribuicaoespacial-da-populacao-segundocor-ou-raca-pretos-epardos. html?edicao $=16125 \& \mathrm{t}=\mathrm{sobr}$ e. Acesso em 02 Jun 2019.

19. Zajonz D, Behrens J, Brand AG, Hoch A, Fakler JKM, Roth A, et al. Femoral neck fractures in old age treated with hemiarthroplasty. Der Orthopade. 2019; 48(3):224231.

Conflito de interesses: Os autores declaram não haver conflito de interesses.

Participação dos autores:

Concepção: Porto AL, Leal CBM, Rios MA.

Desenvolvimento: Porto AL, Leal CBM, Rios MA.

Redação e revisão: Porto AL, Leal CBM, Rios MA, Fernandes TSS, Fernandes ESF, Ferreira RBS.

Como citar este artigo: Porto AL, Leal CBM, Rios MA, Fernandes TSS, Fernandes ESF, Ferreira RBS. Características sociodemográficas e custo de hospitalizações por fratura de fêmur em idosos na Bahia. J Health NPEPS. 2019; 4(2):297-309. 